respectively. Diarrhoea was the only $\mathrm{AE}$ observed in $\geq 5 \%$ of patients in either group $(19.6 \%$ vs $7.3 \%)$. No clinically meaningful changes in opioid withdrawal scores and pain intensity were observed in both groups. In EXT, 107 patients completed a 12 week treatment with naldemedine $0.2 \mathrm{mg}$ QD, and the safety profile was similar to that in DBT.

Conclusions Naldemedine improved the symptoms of OIC and was generally well tolerated.

\section{0-10 AN OBSERVATIONAL STUDY OF THE PREVALENCE OF VIVID DREAMS, NIGHTMARES AND SLEEP/NIGHT TERRORS IN PATIENTS WITH ADVANCED CANCER AND THEIR ASSOCIATION WITH OPIOD ANALGESICS}

Shuchita Patel, Andrew Davies. Royal Surrey County Hospital, Guildford, UK

\subsection{6/bmjspcare-2017-00133.10}

Aim The aim of this study was to investigate vivid dreams, nightmares and sleep/night terrors in patients with advanced cancer.

Methods The study was a multicentre, prospective observational study. Single interviews were conducted with 174 patients and data were collected on their demographics, cancer diagnosis, co-morbidities, current medication and patient's assessment of the ECOG performance status. In addition data were collected on the frequency of vivid dreams, nightmares and sleep/night terrors, as well as the patient's sleep quality and physical and psychological symptoms (Memorial Symptom Assessment Scale and Pittsburgh Sleep Quality Index).

Results Sixty (34\%) patients reported vivid dreams, 31 (18\%) patients reported nightmares and $14(8 \%)$ patients reported sleep/night terrors. Vivid dreams were associated with presence of psychological symptoms, but not physical symptoms $(p=0.315)$. Nightmares were associated with presence of both physical and psychological symptoms. None of these phenomena were associated with the use of opioid analgesics.

Conclusion Vivid dreams are relatively common in patients with advanced cancer, although nightmares and sleep/night terrors occur less frequently in this population (and no more frequent than in the general population). Vivid dreams appear to be primarily associated with psychological problems, and so patients reporting these should be screened for psychological problems. Similarly, patients with nightmares should be screened for psychological problems, and have their physical symptoms adequately controlled.

\section{Poster Presentations}

\section{P-11 WHAT DO END STAGE RESPIRATORY DISEASE PATIENTS GET FROM HOSPICE SERVICES?}

\footnotetext{
1,2Declan Cawley, ${ }^{1}$ Pauline Dand, ${ }^{1}$ Andrew Thorns. ${ }^{1}$ Pilgrims Hospices, Canterbury, UK; ${ }^{2}$ University Of Kent, UK
}

10.1136/bmjspcare-2017-00133.11

Background Non-malignant respiratory diseases (NMRD) are increasing in incidence and prevalence with figures rises with our globally ageing population. This coupled with multimorbidity is likely to increase the needs of individuals from a supportive and palliative care approach. The challenge within fiscally constraint health economies, is to ensure equity of care across all care settings so the individual gets care of an expected standard rather than duplication or omissions within the current services delivering the care.

Aim To explore how patients with non-malignant respiratory diseases traverse through the hospice organisation and how consistent is this approach across 3 hospice sites.

Method A retrospective case note of review of patients referred with NMRD to a hospice organisation within 1 year.

Results 169 case notes were identified with a convenient sample of 100 explored for further analysis. Length of contact varied for days to months (18) with median being 30-90 days. The majority of patients (97) had COPD, were Males (59) with median age 78 years. 63 patients had multi-morbidity $(>2)$ with Heart Failure, IHD and Cancer being the most common. Only 60 cases had an identified carer with 50\% having external professional help. Breathlessness (81) and anxiety (34) were the common presenting symptom with the vase majority of patients having a formal holistic clinical review (60), medication review (65) and attendance at a breathlessness management group (58). Opioids were commonly taken (57) along with benzodiazepines (58). Advance care planning (ACP) was attempted in the majority of cases with DNAR (63), PPOD (49) with only 15 cases explicitly reporting ceilings of care.

Conclusions Hospice care and the need for supportive and palliative care needs to dovetail with existing services and articulate clearly what and when it intends to provide input. Prognostic uncertainty, awareness and parallel planning for EOLC requires a whole systems approach.

\section{P-12 USE OF ANXIOLYTIC AND ANTIPSYCHOTIC MEDICATIONS IN THE DYING PHASE AMONGST HOSPICE INPATIENTS}

Amy Hawkins, Emily Sills. Woking and Sam Beare Hospices, Weybridge, UK

\subsection{6/bmjspcare-2017-00133.12}

Background Terminal agitation and delirium at the end of life are common. ${ }^{1}$ Whilst anxiolytic and antipsychotic medications are widely used during the last week of life, clinical evidence regarding their use is limited. ${ }^{2,3}$ Our aim was to audit current practice at two inpatient units.

Methods A retrospective audit was carried out of all patients who died during a four-month period (June to October 2015) at two hospices $(n=75)$. Data were collected on whether anxiolytic and/or antipsychotic medications were used in the last week of life, the $\operatorname{drug}(\mathrm{s})$ and $\operatorname{dose}(\mathrm{s})$ administered, and the indication. Use of Levomepromazine and Haloperidol for nausea and vomiting were excluded. Audit standards were set according to guidance in the PCF- $5^{4}$ and a compliance target of $80 \%$ was set.

Results The median age was 79 years (range 32 to 94) and $80 \%$ of patients had a primary diagnosis of cancer. In total, 91.7\% $(n=33)$ of patients at Hospice $1 \%$ and $82.1 \%(n=32)$ at Hospice 2 received anxiolytic and/or antipsychotic medications during the last week of life. The most common indication at Hospice 2 was terminal agitation $(n=16,50 \%)$, compared with mixed terminal agitation and delirium $(n=11$, $33.3 \%$ ) at Hospice 1. Midazolam was the most widely used drug (used in $77.8 \%$ of patients at Hospice $1, \mathrm{n}=28$, and $74.4 \%$ of patients at Hospice 2, $n=29$ ). Haloperidol was more widely used at Hospice 1 whereas Levomepromazine 